Published in final edited form as:

Eat Disord. 2015 ; 23(4): 356-365. doi:10.1080/10640266.2015.1044349.

\title{
Couple-Based Interventions for Adults with Eating Disorders
}

\author{
JENNIFER S. KIRBY*, \\ Department of Psychiatry, University of North Carolina at Chapel Hill, USA \\ CRISTIN D. RUNFOLA*, \\ Department of Psychiatry, University of North Carolina at Chapel Hill, USA \\ MELANIE FISCHER, \\ Department of Psychology, University of North Carolina at Chapel Hill, USA \\ DONALD H. BAUCOM ${ }^{\star \star}$, and \\ Department of Psychology, University of North Carolina at Chapel Hill, USA \\ CYNTHIA M. BULIK ${ }^{*}$ \\ Department of Psychiatry, Department of Nutrition, University of North Carolina at Chapel Hill, \\ USA; Department of Medical Epidemiology and Biostatistics, Karolinska Institutet, Stockholm, \\ Sweden
}

\section{Abstract}

\begin{abstract}
A significant number of adults with eating disorders fail to achieve relief from the disorder, with many dropping out from treatment or relapsing. Standard treatment remains individual therapy despite partners being negatively affected and typically wanting to help in an effective and loving way. We propose that couple-based interventions, which leverage the support of a partner and the relationship in treatment, may improve outcome and recovery rates for adults with eating disorders. In this paper, we survey the empirical literature supporting the treatment of adults in a couple context and describe our existing and emerging couple-based interventions for eating disorders.
\end{abstract}

\section{Couple-Based Interventions for Adults with Eating Disorders}

Since the early 2000s, the landscape for eating disorders treatment of children and adolescents has gradually shifted from a focus on individual to family-based intervention. This shift has been based on two key findings. First, interpersonal factors have been found to play an important role in illness persistence (Arcelus, Haslam, Farrow, \& Meyer, 2013; Goddard et al., 2011). Second, including family members in the treatment of adolescents with eating disorders has improved outcome (Lock, 2015). Whereas marked shifts in the care of adolescent patients have yielded positive benefits, typical treatment for adults with

\footnotetext{
Correspondence: Donald H. Baucom, CB\# 3270, Department of Psychology, UNC, Chapel Hill, NC 27599-3270, donbaucom@unc.edu; or Cynthia M. Bulik, CB\#7160, Department of Psychiatry, 101 Manning Drive, UNC, Chapel Hill, NC 27599-7160, cbulik@med.unc.edu.

* Kirby and Runfola contributed equally to this manuscript and share the role of first author.

** Baucom and Bulik are co-directors of the UCAN/UNITE treatment program and contributed equally to this manuscript.

Declaration of Interest: Dr. Bulik is a consultant and grant recipient from Shire Pharmaceuticals.
} 
eating disorders has remained individual therapy and outcomes, which are modest at best, have stagnated (Berkman et al., 2006; Brown \& Keel, 2012). To help improve outcome and recovery rates for adults with eating disorders, we created couple-based interventions that incorporate the partner into treatment in a developmentally appropriate and evidenceinformed manner. In this paper, we survey the empirical literature supporting the treatment of adults in a couple context and describe our existing and emerging couple-based interventions for eating disorders.

\section{Why Couple-Based Treatments for Eating Disorders?}

Individuals who have recovered from eating disorders describe supportive relationships as vital to their recovery (Linville, Brown, Sturm, \& McDougal, 2012; Tozzi, Sullivan, Fear, McKenzie, \& Bulik, 2003). Contrary to popular opinion, adults with eating disorders appear to enter into committed relationships at a rate comparable to healthy peers (Maxwell et al., 2010). However, some may struggle with maintaining healthy relationships in the midst of an eating disorder as the disorder is taxing on not only the individual, but also the partner and the relationship. Couples with eating disorders report significant relationship distress, reduced levels of positive interaction, and more negative communication than couples without eating disorders (Van den Broucke, Vandereycken, \& Vertommen, 1995a; Woodside, Lackstrom, \& Shekter-Wolfson, 2000; Whisman, Dementyeva, Baucom, \& Bulik, 2012). Difficulties around sexual functioning and intimacy in the relationship are also common (Pinheiro et al., 2010; Van den Broucke, Vandereycken, \& Vertommen, 1995b).

Challenges within the romantic relationship are likely due to many patient and partner factors (including biological, temperamental, behavioral, and environmental) related and unrelated to the disorder. For example, many individuals with anorexia nervosa (AN) are emotionally avoidant and struggle to express their feelings, which may negatively affect their ability to articulate their needs, tolerate distress in the relationship, or remain close with others (Schmidt \& Treasure, 2006; Van den Broucke et al., 1995a). Adults with bulimia nervosa (BN) generally lack constructive communication skills and tend to be impulsive (Van Buren \& Williamson, 1988; Van den Broucke et al. 1995a), which may contribute to negative interactions within the relationship. Individuals with BED experience emotions intensely and have been described as having difficulties with boundary setting and over involvement in relationships, which may contribute to their experience of interpersonal relationships as stressful (Riener, Schindler, \& Ludvik, 2006). Characteristic of eating disorders is body distress and shame, which likely contributes to a patient's anxiety around exposing his/her body to partners and engaging in intimate acts. Moreover, hormonal changes in the context of eating disorders results in reduced libido and, thus, interest in sex, which can be confusing to the couple.

Partners also struggle relative to the eating disorder and within the relationship. Partners commonly report difficulty understanding the eating disorder and finding the secrecy surrounding eating disorder behaviors challenging to live with (Huke \& Slade, 2006). Many describe feelings of powerlessness and as though their well-intentioned attempts at supporting their loved one backfire. Some partners become fearful of saying or doing something hurtful or counterproductive and, thus, become avoidant whereas others become 
critical or blaming. This behavior can inadvertently support or exacerbate the patient's shame, secrecy, and self-critical nature. Partners often want to help but do not know what to do. These challenges can cause caregiver distress and burden (Fischer, Baucom, Kirby, \& Bulik, 2015; Zabala, Macdonald, \& Treasure, 2009), and may contribute to escalating conflict or issues in the relationship.

Such distress and conflict within the relationship is noteworthy as a distressed, critical, or hostile relationship has been shown to elevate the risk for illness persistence and relapse in various psychiatric disorders (Baucom, Belus, Adelman, Fischer, \& Paprocki, 2014). Fortunately, cognitive-behavioral couple therapy (CBCT; Epstein \& Baucom, 2002) that targets interpersonal dynamics to enhance relationship satisfaction, has demonstrated effectiveness with treating a variety of psychiatric disorders, including two highly comorbid with eating disorders-depression and anxiety (Baucom, et al., 2014). These data, which suggest that couple-based interventions for eating disorders may help more effectively treat the disorder, lead us to develop the first manualized couple-based intervention for AN.

\section{Uniting Couples in the Treatment of Anorexia Nervosa (UCAN)}

UCAN (Bulik, Baucom, Kirby, \& Pisetsky, 2011) integrates CBCT and cognitivebehavioral therapy (CBT) for AN to treat the disorder. Given the complexity of AN, UCAN was designed as an augmentation to individual therapy, nutritional counseling, and medication management to ensure treatment appropriately addresses all aspects of the disorder. Unlike family-based treatment with children and adolescents (Lock, Le Grange, Agras, \& Dare, 2001), which initially provides significant others (i.e., parents) with control over the re-nourishment and recovery process, UCAN works with loved ones (i.e., couples) to reach joint decisions regarding treatment and recovery from AN. In this way, the partner is incorporated into treatment as a partner of equal status in a developmentally appropriate manner.

UCAN is believed to benefit couples by facilitating three broad mechanisms of change, two focal to AN and one addressing broader relationship functioning. First, the UCAN therapist works to create conditions that allow the secrecy and avoidance commonly associated with AN to be addressed and to decrease (mechanism 1). Bringing AN into the open enables the patient to receive support from the partner, enhances understanding of illness, and helps to increase patient accountability. Partners are kept informed throughout treatment about the patient's progress and are actively involved in team discussions regarding the need for higher levels of care. Critically, at times when patients are struggling and motivation to stay in treatment decreases, the partner and the relationship can be "leveraged" to keep the patient engaged in treatment. UCAN also brings partners into treatment more specifically by helping the couple work as a team to address AN (mechanism 2). Throughout treatment, the couple is taught how to work effectively against the AN including addressing mealtimes, responding when a patient feels the urge to restrict or purge, or developing strategies for social outings or holidays. Third, as described above, AN and relationship difficulties can negatively influence one another; a distressed relationship can be a source of chronic stress for the individual with AN, and AN places considerable strain on the relationship. 
Therefore, to reduce stress on the patient and facilitate recovery from AN, we address overall relationship functioning within UCAN when needed (mechanism 3).

\section{UCAN Treatment}

\section{Phase One: Creating a Foundation for Later Work}

The UCAN treatment begins by helping the couple build a supportive foundation for addressing AN effectively as a team by targeting three goals: (a) understanding the couple's experience of $\mathrm{AN}$, (b) providing psychoeducation about AN and the recovery process, and (c) teaching the couple communication skills. These treatment procedures aim to reduce the secrecy and avoidance surrounding AN and build the couple's ability to communicate effectively; thus, all three mechanisms described above are in play from the outset of UCAN.

Assessment and psychoeducation-UCAN begins with a thorough assessment of the couple's relationship, AN symptoms, and AN associated features. Each AN symptom (e.g., low weight, fear of weight gain, restricting) is presented to the couple, and both partners share their experiences and observations of each characteristic. This same process is conducted for comorbid conditions, such as depression, anxiety, substance abuse, etc. The therapist then discusses biological and environmental risk and maintenance factors for AN, as well as details about the recovery process, addressing any questions or misconceptions either partner may have. By discussing this psychoeducation material with both members of the couple, UCAN decreases the secrecy around AN and works to create a comprehensive and shared understanding of AN to help foster the couple's teamwork.

Communication training-Phase one of UCAN concludes with teaching the couple communication skills to help them share their thoughts and feelings with each other, along with strategies for making sound decisions. Through didactic instruction and extensive inand out-of-session practice, the couple learns how to express thoughts and feelings, listen responsively, and solve problems/make decisions as a team. More specifically, the emotional expressiveness skills focus on couples sharing their personal experiences in a subjective and specific manner, including their emotions related to the AN, one another, and the relationship. These conversations facilitate the couple taking risks in terms of being more open and vulnerable. The couple is taught how to respond to such disclosures using active listening skills. These listening skills help partners demonstrate that they have heard one another accurately, and that they accept each other's experiences even if they do not agree. This distinction between "acceptance and agreement"- that it is possible to accept one another's experience as being valid and authentic, and yet not have to agree-is paramount for couples experiencing AN. Frequently, the patient and partner have different perspectives relative to the disorder with some of these having significant implications, such as whether the patient is indeed ill, underweight, or needs a higher level of care. The emotional expressiveness skills create a way for couples to have such conversations, so that both partners feel understood even when their perspectives differ. Couples are also taught how to make decisions together by first articulating what is important to each partner and then working to honor these needs and compromise toward a mutually agreed upon decision. 
The above skills are applied in couple therapy across a broad set of issues, but in UCAN, the application of these skills involves couples' conversations about the AN. Couples also are encouraged to apply these skills outside of session to issues within their general relationship, so they may also contribute to improvements in the couple's overall relationship quality.

\section{Phase Two: Addressing Anorexia Nervosa Within a Couples Context}

Resuming a healthy body weight and developing healthy eating behaviors (e.g., avoidance of restricting and purging) are major treatment goals for individuals with AN. Phase two, the majority of the UCAN treatment, parallels the patient's efforts in individual therapy by concurrently helping the couple develop a good support system for this individual work.

Addressing eating disordered behavior-In phase two, the couple works together to address a series of AN challenges to foster healthful eating, exercise, and other relevant behavioral patterns, including arranging to eat together, addressing concerns around eating in public, and changing unhelpful couple interaction patterns towards more appropriate strategies. With the help of their UCAN therapist, the couple is guided in a thoughtful consideration of AN features they find most challenging, and then how to employ their communication skills in responding to these challenges more successfully together. Consistent with exposure principles of working toward more challenging behaviors over time, the couple selects a lower level AN behavior to address first, such as creating a more positive family mealtime environment. Regarding mealtimes, both patients and partners commonly describe feeling anxious and worrying what the other is thinking or may do. The patient may worry that the partner is being a "food police," and the partner may be frightened that the patient is not eating enough or unsure what to say or do with this concern. The therapist guides the couple in developing specific strategies to help mealtimes be more calm and conducive to healthy eating (e.g., creating a regular schedule for meals, or a plan for what to discuss and not discuss during meals).

Body image concerns-Phase two of UCAN continues by broadening the AN focus to body image and physical intimacy issues. Applying their communication skills, the couple discusses body image issues and how they can better interact around this challenging domain. Body image distortions and body dissatisfaction can be two of the most confusing and challenging features of AN for the partner; therefore, it is important that the couple has an opportunity to increase understanding and empathy for each other's body image experiences. Once they have a greater appreciation of how each partner experiences this domain, the couple can use their decision-making skills to create supportive ways of interacting around body image that are conducive to recovery. For example, the couple may decide that the (female) patient will describe to the partner how she feels about her body without focusing on "being fat," and the partner will practice using active listening to express his understanding of her feelings and offer a hug provided the patient finds physical closeness to be supportive. By using the emotional expressiveness skills of the patient being subjective and specific and the partner communicating that he accepts (without having to agree) the patient's distress, the couple is better able to discuss this topic, helping both parties to feel more supported and understood. 
Physical intimacy and affection-Building from the body image domain, phase two concludes with a consideration of the couple's physical intimacy relative to AN. The therapist discusses how sex and physical affection can affect and be affected by the experience of negative body image and an eating disorder, incorporating the couple's specific experiences throughout this discussion. Included in this exploration is how the couple experiences challenges within their physical relationship more broadly as well. Because couples vary widely in their physical and sexual relationships, UCAN is tailored to the patient's and couple's current level of functioning and assists them in developing healthier patterns within their physical relationship. For example, couples who have not been physically close may first work on discussing the possibility of physical touch or beginning to hold hands. Other couples who continue to have physical closeness may focus on building greater quality or quantity of physical intimacy in their lives.

\section{Phase Three: Relapse Prevention and Termination}

Phase three of UCAN brings the treatment to a close, focusing on relapse prevention and the couple's next steps in the AN recovery process. The therapist provides psychoeducation on recovery and relapse prevention to help ensure that the couple has realistic, shared expectations for the nonlinear recovery typical of AN. With the therapist's guidance, the couple develops appropriate agreed-upon responses should a slip or full relapse occur for both (a) the patient's eating-disordered behaviors and related symptoms, and (b) the couple's approach to addressing the disorder. Thus, the couple might address how they will discuss their concerns if the patient returns to restricted eating, rather than avoiding the issues as a couple. The treatment concludes with a review of the UCAN experience and a consideration of how the couple needs to continue working together toward recovery from AN.

\section{UCAN Initial Observations}

The UCAN program is currently undergoing extensive evaluation with couples in committed relationships. However, results from the pilot trial (Bulik \& Baucom, 2012) are highly encouraging, and clinical observations suggest that including partners in the treatment process is of considerable value. First, partners helped keep patients in treatment-only 5\% of patients dropped out from our initial UCAN trial in comparison to the $~ 25-40 \%$ drop out rate observed in previous clinical trials of medication and behavioral interventions for AN (Berkman et al., 2006). Second, UCAN resulted in much greater weight gain over the course of treatment than other clinical interventions $\left(M_{\mathrm{BMI}}\right.$ increase of $2.9 \mathrm{vs} .1 \mathrm{~kg} / \mathrm{m}^{2}$; Berkman et al., 2006) suggesting that it was more effective at restoring the patient to physical health. Third, psychological functioning also improved for UCAN patients, with eating disorder symptoms reducing in severity. Fourth, the couples reported experiencing improvements in their relationship functioning. Thus, preliminary data suggest that the UCAN program improves outcome for adults with AN. Since the development of UCAN, case reports of adult patients with eating disorders receiving couple therapy to augment their individual therapy have been published and show promise as well (Murray, 2014; Reyes-Rodriguez, Baucom, \& Bulik, 2015). 


\section{Extension of UCAN to Other Eating Disorders (UNITE)}

The benefit of UCAN in AN recovery naturally raised the question of whether couple-based interventions could also be of benefit for patients and partners affected by other eating disorders-namely, BN and BED. Thus far, we have designed a couple-based intervention for BED that builds from the UCAN foundation and is tailored to the specific features of BED. UNITE-BED tackles delicate issues such as how weight is discussed by the couple, including the role of weight stigma; encourages couples to focus on health rather than weight; and addresses fundamental misunderstandings regarding the nature of BED as an illness, not a choice. This program is undergoing pilot testing, with recruitment data thus far suggesting couples are very interested in the program.

If proven effective, these couple-based programs (UCAN and UNITE) have the potential to transform clinical practice for adults with eating disorders who are in relationships. We aim to develop readily accessible dissemination platforms that will enable training of therapists around the world in these promising interventions.

\section{Acknowledgments}

Financial Support: This research was supported in part by grants MH082732-01 and MH093615 from the National Institute of Mental Health to Cynthia M. Bulik and Donald H. Baucom, MPIs. Cristin D. Runfola was supported by the Global Foundation for Eating Disorders (www.GFED.org; PIs: Bulik and Baucom). UNITE is also supported by a grant from the Hilda and Preston Davis Foundation. The project described was supported by the National Center for Research Resources and the National Center for Advancing Translational Sciences, National Institutes of Health, through Grant Award Number UL1TR000083. The content is solely the responsibility of the authors and does not necessarily represent the official views of the NIH.

We would like to thank Emily Pisetsky, Kathryn Nowlan, Camden Matherne, Robert Hamer, Brian Baucom, Jennifer Belus, and McKenzie Roddy for their contributions to this research.

\section{References}

Arcelus J, Haslam M, Farrow C, Meyer C. The role of interpersonal functioning in the maintenance of eating psychopathology: A systematic review and testable model. Clinical Psychology Review. 2013; 33(1):156-167. [PubMed: 23195616]

Baucom DH, Belus JM, Adelman CB, Fischer MS, Paprocki C. Couple-based interventions for psychopathology: A renewed direction for the field. Journal of Family Therapy. 2014; 53(3):445461.

Berkman, ND.; Bulik, CM.; Brownley, KA.; Lohr, KN.; Sedway, JA.; Rooks, A.; Gartiehner, G. Evid Rep Technol Assess. Rockville, MD: Agency for Research and Quality; 2006. Management of eating disorders. Evidence Report/Technology Assessment No. 135 (Prepared by the RTI International-University of North Carolina Evidence-Based Practice Center under Contract NO. 290-02-0016.) AHRQ Publication NO. 06-E010.

Brown TA, Keel PK. Current and emerging directions in the treatment of eating disorders. Substance Abuse. 2012; 6:33-61. [PubMed: 22879753]

Bulik, CM.; Baucom, DH. Uniting Couples (in the treatment of) Anorexia Nervosa (UCAN). Workshop presented at the International Conference on Eating Disorders; Austin, TX. 2012.

Bulik CM, Baucom DH, Kirby JS, Pisetsky E. Uniting Couples (in the treatment of) Anorexia Nervosa (UCAN). International Journal of Eating Disorders. 2011; 44(1):19-28. [PubMed: 20063308]

Epstein, NB.; Baucom, DH. Enhanced Cognitive-Behavioral Therapy for Couples:A Contextual Approach. Washington, D.C: American Psychological Association; 2002. 
Fischer MS, Baucom DH, Kirby JS, Bulik CM. Partner distress in the context of adult anorexia nervosa: The role of patients' perceived negative consequences of AN and partner behaviors. International Journal of Eating Disorders. 2015; 48(1):67-71. [PubMed: 25087574]

Goddard E, Macdonald P, Sepulveda AR, Naumann U, Landau S, Schmidt U, Treasure J. Cognitive interpersonal maintenance model of eating disorders: Intervention for carers. The British Journal of Psychiatry. 2011; 199(3):225-231. [PubMed: 21727233]

Huke K, Slade P. An exploratory investigation of the experiences of partners living with people who have bulimia nervosa. European Eating Disorders Review. 2006; 14(6):436-447.

Linville D, Brown T, Sturm K, McDougal T. Eating disorders and social support: Perspectives of recovered individuals. Eating Disorders. 2012; 20(3):216-231. [PubMed: 22519898]

Lock J. An update on evidence-based psychosocial treatments for eating disorders in children and adolescents. Journal of Clinical Child \& Adolescent Psychology. 2015; 0(0):1-15. [PubMed: 25945418]

Lock, J.; Le Grange, D.; Agras, WS.; Dare, C. Treatment manual for anorexia nervosa: A family-based approach. New York: Guilford Publications, Inc; 2001.

Maxwell M, Thornton LM, Root TL, Pinheiro AP, Strober M, Brandt H, Bulik CM. Life beyond the eating disorder: Education, relationships, and reproduction. International Journal of Eating Disorders. 2010; 44(3):225-232. [PubMed: 20143323]

Murray SB. A case of strategic couples therapy in adult anorexia nervosa: The importance of systems in context. Contemporary Family Therapy. 2014; 36:392-397.

Pinheiro AP, Raney TJ, Thornton LM, Fichter MM, Berrettini WH, Goldman D, Bulik CM. Sexual functioning in women with eating disorders. International Journal of Eating Disorders. 2010; 43(2):123-129. [PubMed: 19260036]

Reyes-Rodriguez ML, Baucom DH, Bulik CM. Culturally sensitive intervnetion for Latina women with eating disorders: A case study. Mexican Journal of Eating Disorders. 2015; 5:135-145.

Riener R, Schindler K, Ludvik B. Psychosocial variables, eating behavior, depression, and binge eating in morbidly obese subjects. Eating Behavior. 2006; 7(4):309-314.

Schmidt U, Treasure J. Anorexia nervosa: Valued and visible. A cognitive-interpersonal maintenance model and its implications for research and practice. British Journal of Clinical Psychology. 2006; 45(Pt 3):343-366. [PubMed: 17147101]

Tozzi F, Sullivan PF, Fear JL, McKenzie J, Bulik CM. Causes and recovery in anorexia nervosa: The patient's perspective. International Journal of Eating Disorders. 2003; 33(22):143-154. [PubMed: 12616580]

Van Buren DJ, Williamson DA. Marital relationships and conflict resolution skills of bulimics. International Journal of Eating Disorders. 1988; 7(6):735-741.

Van den Broucke S, Vandereycken W, Vertommen H. Marital communication in eating disorder patients: A controlled observational study. International Journal of Eating Disorders. 1995a; 17(1): 1-21. [PubMed: 7894447]

Van den Broucke S, Vandereycken W, Vertommen H. Marital intimacy in patients with an eating disorder: A controlled self-report study. British Journal of Clinical Psychology. 1995b; 34(Pt 1): 67-78. [PubMed: 7757040]

Whisman MA, Dementyeva A, Baucom DH, Bulik CM. Marital functioning and binge eating disorder in married women. International Journal of Eating Disorders. 2012; 45(3):385-389. [PubMed: 21560137]

Woodside DB, Lackstrom JB, Shekter-Wolfson L. Marriage in eating disorders comparisons between patients and spouses and changes over the course of treatment. Journal of Psychosomatic Research. 2000; 49(3):165-168. [PubMed: 11110987]

Zabala MJ, Macdonald P, Treasure J. Appraisal of caregiving burden, expressed emotion and psychological distress in families of people with eating disorders: A systematic review. European Eating Disorders Review. 2009; 17(5):338-449. [PubMed: 19367608] 